Variations tibétaines, Et autres...

\title{
PANG Tatjana, STARY Giovanni, Manchu versus Ming: Qing Taizu Nurhaci's "Proclamation" to the Qing Dynasty
}

Wiesbaden, Harrassowitz in Kommission (Aetas Manjurica 14), 2010, xv, 68 p. ISBN 9783447061605

Natalie Köhle

\section{(2) OpenEdition} Journals

Édition électronique

URL : https://journals.openedition.org/emscat/1919

DOI : 10.4000/emscat.1919

ISSN : 2101-0013

Éditeur

Centre d'Etudes Mongoles \& Sibériennes / École Pratique des Hautes Études

Référence électronique

Natalie Köhle, "PAng Tatjana, Stary Giovanni, Manchu versus Ming: Qing Taizu Nurhaci's

"Proclamation" to the Qing Dynasty », Études mongoles et sibériennes, centrasiatiques et tibétaines [En ligne], 42 | 2011, mis en ligne le 20 décembre 2011, consulté le 13 juillet 2021. URL : http:// journals.openedition.org/emscat/1919; DOI : https://doi.org/10.4000/emscat.1919 
Pang, Tatjana and Giovanni Stary, Manchu versus Ming: Qing Taizu Nurhaci's "Proclamation" to the Qing Dynasty (Wiesbaden, Harrassowitz in Kommission) (Aetas Manjurica 14), 2010, xv, 68 pp. ISBN 9783447061605

Par Natalie Köhle

Cet ouvrage offre une transcription et une traduction de la xylographie mandchoue "Guimet 61626 », avec une réimpression et une traduction de la version chinoise de ce texte communément connu en tant que «Proclamation of the Latter Jin to the Ming Wanli Emperor »Hou Jin xi Ming Wanli huangdi wen 後金檄明萬曆皇帝文 (ni le chinois ni l'original mandchou ne portent de titre).

Composé entre 1623 et 1626, pendant la conquête mandchoue des territoires chinois dans le Liaodong, ces documents offrent dix-neuf épisodes ou « exemples » de l'histoire chinoise ancienne et de l'histoire contemporaine mandchoue qui gravitent autour de l'idéologie chinoise ancienne du mandat du Ciel. Ils visent à prouver le droit de Nurhaci à vaincre la dynastie des Ming car elle a perdu son «mandat à régner », c'est-àdire sa légitimité ${ }^{1}$. La présente publication, Manchu versus Ming, est complète avec une introduction, une analyse des nouveaux éléments de preuve concernant la paternité et la datation des documents, ainsi que des commentaires des épisodes ou exemples. Dans une publication antérieure d'une édition facsimile de Guimet 61626 par Pang et Stary $^{2}$, les auteurs avaient proposé que Guimet 61626 pourrait être la version mandchoue de 後金 檄明萬曆皇帝文 Hou Jin xi Ming Wanli huangdi wen ${ }^{3}$. Comprenant une comparaison détaillée de ces documents avec d'autres sources pertinentes contemporaines, comme le

\footnotetext{
${ }^{1}$ Pang Xiaomei 龐曉梅, «Manchu-Chinese text of 'Proclamation of Nurhaci to the Ming' : what version was written fist (sic)? », Qingshi lunji. Qinghe Wang Zhonghan jiaoshou jiushi huadan 清史論集。慶賀 王鍾翰教授九是十華誕, ed. Zhu Chengru 朱誠如 (Pékin, Zijincheng chubanshe, 2003), p. 710.

2 Pang, Tatjana and Giovanni Stary, New Light on Manchu Historiography (Wiesbaden, Harrassowitz, 1998).

${ }^{3}$ Un fac-similé de cette xylographie a été publié par Imanishi Shunjū in 1973 «"Kō Kin geki Min Banreki kōtei bun” ni tsuite» 後金檄明萬歴皇帝について, Chōsen gakuhō, 67 (1937), pp. 137-158; traduit en allemand (sans fac-simile) : "Über einen Aufruf der Späteren Chin an die Ming von ca. 1623 », Oriens Extremus, 20/1 (1973), pp. 27-37. Une réimpression en chinois simplifié a été publiée par Pan Zhe 潘吉, Sun Fangming 孫方明 et Li Hongbin 李鴻彬 (eds) dans Qing ruguan qian shiliao xuanji 清入關前史料 選輯 vol. 1 (Pékin : Zhongguo renmin daxue chubanshe, 1985-<1989>), pp. 289-296.
} 
Jiu Manzhou dang 舊滿洲檔, Daicing gurun-i fukjin doro neihe bodogon-i bithe (Huang Qing kaiguo fanglüe 皇清開國方略) ${ }^{4}$, et une copie manuscrite d'une partie de Guimet $61626^{5}$, Manchu versus Ming suit et complète l'étude antérieure de Pang et Stary.

Les nouveaux éléments apportés par cette récente étude viennent compléter, en les révisant, les conclusions antérieures de Pang et Stary. Ils rejettent à présent leurs hypothèses antérieures concernant la paternité du document et proposent que l'auteur du texte soit le scribe appelé Mucengge - plus probablement le mujilen bahabukū et hash $\bar{u}$ ergi ashan-i amban du Ministère de la guerre -, qui est mentionné comme un compagnon de la figure littéraire de Dahai dans le Jiи Manzhou dang. Ainsi ils donnent 1623 comme terminus post quem et 1626 comme terminus ante quem pour la composition du manuscrit. Cette période confirme la date de composition donnée comme «vers 1623 », qui avait été proposée en 1973 par Imanishi Shunjū. Pang et Stary voient ces manuscrits comme des « literary compilation(s), based on Nurhaci's utterances as recorded in the Jiu Manzhou dang » plutôt que comme des documents de propagande. Ils soutiennent que l'intention littéraire justifie la composition parallèle des documents en chinois et en mandchou, ce qui n'aurait pas été nécessaire s'il s'était agi de propagande.

Naturellement, la traduction que proposent Pang et Stary du récit d'un tel témoin du début de l'histoire textuelle mandchoue est une précieuse addition au corpus d'études mandchoues. Cette publication vise à être un volume d'accompagnement au New Light on Manchu Historiography et, comme telle, elle est très utile. Cependant, sans avoir sous la main le New Light on Manchu Historiography, Manchu versus Ming est assez difficile à utiliser. Sans cet ouvrage de référence en effet, on ne peut comprendre le contexte historique et textuel, car l'introduction des documents traduits dans Manchu versus Ming est minime et semble présupposer une familiarité avec les publications antérieures de Pang et Stary à ce sujet. Par exemple, dans l'introduction, les auteurs comparent Guimet 61626 avec Guimet 61625 sans fournir au lecteur d'informations sur Guimet 61625 autre qu'une référence à New Light on Manchu Historiography ${ }^{6}$.

\footnotetext{
${ }^{4}$ Xylographie de 1789. Cet œuvre a été traduite en allemand par Erich Hauer, Huang-Ts'ing K'ai-kuo Fang-lüeh. Die Gründung des mandschurischen Kaiserreiches (Berlin et Leipzig : W. de Gruyter, 1926).

${ }^{5}$ Publié sous le titre «Lao manwen shangyu » 老满上谕文 dans Congbian wenxian, 2 (1937), réimprimé à Taipei, 1964, vol. shang p. 82, vol. xia pp. 1053-1054 (voir p. xiii de la publication en revue).

${ }^{6}$ Voir p. $\mathrm{x}$ et $\mathrm{p}$. xv de la publication en revue.
} 
En ce qui concerne l'analyse des manuscrits, Pang et Stary observent des différences entre les deux versions, mais là aussi l'analyse de ces différences est réduite au minimum. Il aurait été souhaitable d'accéder à une analyse plus approfondie des différences linguistiques entre les versions mandchoue et chinoise du document : quelles différences ont été introduites expressément pour rejoindre des auditoires culturellement et politiquement différents ? Qu'impliquent les choix de traduction des Mandchous sur les débuts de la lexicographie et de l'histoire littéraire mandchoue ?

Heureusement, une telle analyse du contexte historique et des différences entre les versions mandchoue et chinoise se trouve dans une excellente contribution de Pang intitulée «Manchu-Chinese text of 'Proclamation of Nurhaci to the Ming' : what version was written fist (sic) » publiée en $2003^{7}$. Cependant, puisque l'article de Pang n'est peutêtre pas aussi facilement accessible aux lecteurs que New Light on Manchu Historiography, l'inclusion du contenu de cet article dans l'ouvrage aurait été souhaitable, surtout pour les passages où les auteurs ont rejeté leur choix antérieurs de traduction. Dans le passage suivant, par exemple (reproduction et traduction juxtaposées des passages mandchou et chinois, reproduites de Manchu versus Ming, p. 16) ${ }^{8}$ :

\begin{tabular}{|c|c|c|c|}
\hline [Man.] & [translation from Man.] & [translation from Chin.] & [Chin.] \\
\hline $\begin{array}{l}\text { tūibei tu bithe de jugiyai } \\
\text { han be sirarangge emu } \\
\text { niyalma beri jafabi } \\
\text { ilihabi serengge: } \\
\text { acabume araci jūšen } \\
\text { sere hergen wakao: }\end{array}$ & $\begin{array}{l}\text { In the book Tūibei tu is } \\
\text { said: " A man, after } \\
\text { having taken a bow and } \\
\text { having founded } \\
\text { dynasty] will succeed } \\
\text { the Emperor of the Ju } \\
\text { family". } \\
\text { A Jušen script did not } \\
\text { exist when this [book] } \\
\text { was written! }\end{array}$ & $\begin{array}{l}\text { The Tuibei tu says: "A } \\
\text { f................ bow, } \\
\text { founded [a dynasty]". } \\
\text { A Yi script did not exist, } \\
\text { didn't it? }\end{array}$ & $\begin{array}{l}\text { 推背圖云 } \\
\text { 一【】【】 } \\
\text { 弓立 } \\
\text { 岂非夷字 } \\
\text { 耶 }\end{array}$ \\
\hline
\end{tabular}

\footnotetext{
${ }^{7}$ Pang, « Manchu-Chinese text of 'Proclamation of Nurhaci to the Ming' ».

${ }^{8}$ Note des éditeurs : ne possédant pas la police de caractères adéquate, nous avons remplacé la lettre " $\mathrm{y}$ » surmontée d'un demi-cercle employée par les auteurs de l'ouvrage par un «y » normal.
} 
Cette traduction diffère considérablement de la traduction de Pang 2003. Une référence à cette première traduction aurait été souhaitable. Avec la suggestion, formulée dans Pang 2003, de reconstruire les deux caractères chinois manquants comme [人] et [以] ${ }^{9}$, je suis tentée de lire ce passage en chinois comme « Doesn't 'a man who is holding a bow' refer to the character "barbarian” (yi 夷) ?» Il semble que le sens de ce passage en chinois corresponde à celui du passage parallèle en mandchou, que je préfère lire « it is said that he who will inherit the Zhu family is a man who stands, holding a bow. If we write the two of them together jointly (acabume araci), isn't it the character 'jüšen' (i.e. yi 夷)? ». Si cette traduction du passage mandchou est correcte, elle semble indiquer que la version mandchoue non seulement suit exactement le chinois, mais encore qu'elle cherche à fournir une explication plus détaillée pour le lecteur du mandchou que celle-ci : le caractère $y i$ 夷 (jūšsen) ressemble à un homme debout 人 tenant un arc 弓 - une explication inutile pour les lecteurs de la version chinoise de ce document.

D'autres sections de l'ouvrage discuté dans ce compte-rendu comportent des passages susceptibles d'être lus différemment, en établissant une correspondance plus étroite entre les versions mandchoue et chinoise. Le passage suivant par exemple (Manchu versus Ming, p. 21):

\begin{tabular}{|l|lr|lr|l|}
\hline gūrun-i ejen be gamabi & [But] & when & the & But in which country & 將置主上 \\
wasimbure wara dabala & country's ruler is & will the ruler be & 於何地且 \\
ai obumbi niyalma & captured, he will be & removed? Will the law & 人未有長 \\
bucere & akō & deposed and killed: & of death not be valid for & 生不死之 \\
enteheme banjimbio: & what will happen? Will & not old aged people? & 理 \\
& people who are not & & \\
& killed live forever? & & & \\
\hline
\end{tabular}

Je préfère lire la seconde partie de ce passage comme (Chin.) « There has not yet been such a thing as immortality (長生不死) for men » and (Man.) « Do people not die but live forever? » Autrement dit, dans ce passage, bucere akō enteheme banjimbi semble également être la traduction littérale du chinois 長生不死.

\footnotetext{
9 Pang, « Manchu-Chinese text of 'Proclamation of Nurhaci to the Ming' », p. 713.
} 
Un autre exemple est le passage suivant (reproduit de p. 42 de Manchu versus Ming) :

\begin{tabular}{|c|c|c|c|}
\hline $\begin{array}{l}\text { amban be ajigen obume: } \\
\text { geren be komso obume } \\
\text { gamara be ulhirakō sini } \\
\text { kemuni jase de } \\
\text { sibinjire }{ }^{10} \text { bithe de geli } \\
\text { mini gūrun amban: } \\
\text { cooha geren: simbe } \\
\text { amba alin-i umhan be } \\
\text { gidara gese obumbi } \\
\text { seme hendurengge }\end{array}$ & $\begin{array}{l}\text { Making great things } \\
\text { small, and frequent } \\
\text { things rare, and clear } \\
\text { things unclear, in your } \\
\text { letters sent [?] to my } \\
\text { borderland you always } \\
\text { compared the great } \\
\text { numerous army of my } \\
\text { Dynasty to eggs, which } \\
\text { are crushed by a great } \\
\text { mountain and which } \\
\text { symbolized you. }\end{array}$ & $\begin{array}{l}\text { On purpose you are } \\
\text { cutting great [things] } \\
\text { making them small, } \\
\text { diminishing numerous } \\
\text { [things] making them } \\
\text { few; you also [pretend] } \\
\text { to understand [things] } \\
\text { which you do not know; } \\
\text { with reference to [your] } \\
\text { great Imperial army, } \\
\text { you compare yourself to } \\
\text { a high mountain and me } \\
\text { to a hen's egg, and } \\
\text { foolishly you spoke } \\
\text { about a great mountain } \\
\text { which crushes a hen's } \\
\text { egg. }\end{array}$ & $\begin{array}{l}\text { 故削大為小減眾為寊 } \\
\text { 爾尚不知覺悟仍恃國 } \\
\text { 大兵眾以太山自比以 } \\
\text { 雞卵比我 }\end{array}$ \\
\hline
\end{tabular}

Il semble préférable de lire en mandchou « Making big things small and the many few, you, not understanding things, stated in the communiqués that you kept sending to the border : 'my country is big, [my] troops are many (se référant à la Chine et à l'armée chinoise), [I] will make you (simbe, se référant à Nurhaci) like an egg that is crushed by a big mountain ». On voit que, sauf pour la ligne supplémentaire sur le communiqué kemuni jase de sibinjire bithe de, le mandchou semble suivre le chinois. Cependant, il semblerait que les passages mandchou et chinois se sont mêlés à un passage presque identique, qui suit quelques pages plus loin (Manchu versus Ming, p. 60) :

\footnotetext{
${ }^{10}$ Comme le notent Stary et Pang, le composé sibinjimbi n'est pas attesté dans les principaux dictionnaires mandchous.
} 


\begin{tabular}{|c|c|c|c|}
\hline $\begin{array}{l}\text { gūrun amban: cooha } \\
\text { geren: simbe amba alin- } \\
\text { i umhan be gidara gese } \\
\text { obumbi serengge: }\end{array}$ & $\begin{array}{l}\text { (...) if you say that your } \\
\text { Dynasty is great, your } \\
\text { soldiers are numerous, } \\
\text { that you will be like a } \\
\text { great mountain which } \\
\text { crushes eggs, }[\ldots]\end{array}$ & $\begin{array}{l}(\ldots) \text { if you send } \\
\text { messages interfering } \\
\text { with borderland } \\
\text { [questions], always } \\
\text { repeating that the great } \\
\text { Imperial army will } \\
\text { crush [me] like a great } \\
\text { mountain crushes eggs, } \\
{[\ldots]}\end{array}$ & $\begin{array}{l}\text { 常來插書復說國大兵 } \\
\text { 眾如泰山壓卵等言 }\end{array}$ \\
\hline
\end{tabular}

Ici, la situation est exactement inverse et le détail du communiqué est mentionné dans le passage chinois mais manque dans le passage mandchou correspondant. Ainsi, kemuni jase de sibinjire bithe de du premier passage semble être la traduction littérale de 常来插 書 du second passage.

La comparaison des deux passages ci-dessus (Manchu versus Ming, p. 42 et 60) montre aussi qu'à certains endroits, l'ouvrage aurait bénéficié d'un travail d'édition plus serré, afin d'améliorer la cohérence interne de la traduction. Dans le premier passage, Pang et Stary comprennent « dynastie » et « soldats » en se référant à ceux de Nurhaci, alors que dans le deuxième exemple, ils les interprètent (correctement, à mon avis) comme se référant aux Chinois, même si nous avons affaire à deux passages presque parallèles.

Un autre exemple où un travail d'édition plus approfondi aurait raffermi la cohérence interne de la traduction, est la phrase niyalma buçere akō enteheme banjimbio du deuxième exemple cité ci-dessus (Manchu versus Ming, p. 21), que Pang et Stary traduisent par « Will people who are not killed live forever?» contrairement à une construction similaire qui suit deux pages plus loin fücihi bucerakō enteheme banjimbi sere (Manchu versus Ming, p. 23), traduite «There was a Buddha who was said to be immortal and to live in eternity $\gg$.

Enfin, un véritable travail d'édition n'aurait pas dû laisser dans le texte plusieurs erreurs typographiques (passim), de même que quelques mots mandchous et caractères chinois mal transcrits ou manquants. Par exemple, p. 24, tuwara wara jaka de devrait être tuwara jaka de (p. 33 du fac-simile) ; le quatrième mot de la p. 52 du fac-simile, tondo-i, 
manque dans la transcription (p. 41 de la publication en revue); büton (à la p. 42 de la publication en revue) devrait être būcere ton (p. 53 du facsimile); 平 (à la p. 42 de la publication en revue) devrait être 乎 (16a du ms). Toutefois Manchu versus Ming reste une source précieuse et une traduction d'une grande utilité, car elle rend accessible et juxtapose deux manuscrits rares et importants qui sont des documents pertinents pour l'histoire à la fois du début de la littérature mandchoue et du début du règne des Qing. 